\title{
Concomitant Tricuspid Annuloplasty in Patients Undergoing Totally Endoscopic Mitral Valve Surgery: A Propensity-Score Matched Analysis
}

\author{
Ling-chen Huang, ${ }^{1,2}$ Dao-zhong Chen, ${ }^{1}$ Liang-wan Chen, ${ }^{1}$ Xiao-fu Dai1, ${ }^{2}$ \\ ${ }^{1}$ Department of Cardiovascular Surgery, Union Hospital, Fujian Medical University, Fuzhou, China; ${ }^{2}$ Fujian Key Laboratory of \\ Cardiothoracic Surgery, Fujian Medical University
}

\section{ABSTRACT}

Background: We aimed to evaluate the clinical outcomes of concomitant tricuspid annuloplasty (TAP) in patients undergoing totally endoscopic mitral valve surgery.

Methods: It is a single-center, retrospective study that enrolled a total of 173 patients who underwent mitral valve surgery combined with tricuspid annuloplasty between January 2019 and June 2020 in our institution. Patients who underwent totally endoscopic mitral valve surgery with concomitant tricuspid annuloplasty were categorized into the MIMVS-TAP group $(\mathrm{N}=51)$, and patients who underwent mitral valve surgery with concomitant tricuspid annuloplasty through a median sternotomy were categorized into the MVSTAP group $(\mathrm{N}=122)$. The data collected included detailed demographic and perioperative data. Each patient in the MIMVS-TAP group was individually matched to a patient in the MVS-TAP group, using the propensity scores, and we obtained a matched sample of 51 patients in each group. Parametric and nonparametric tests were used to analyze outcomes.

Results: There were no differences in death rates or related major adverse events between the two groups after propensity score matched analysis. The total operation time was longer in the MIMVS + TAP group versus the MVS+TAP group, as were the mean duration of cardiopulmonary bypass time and the cross-clamp time. The mean duration of intensive care unit stay was longer in the MVS + TAP group compared with that of the MIMVS + TAP group, as was the duration of post-operative hospital stay.

Conclusions: Totally endoscopic mitral valve surgery with concomitant tricuspid annuloplasty can improve a patient's prognosis, with comparable short-term outcomes to those of the open approaches.

\section{INTRODUCTION}

Left-sided valvular heart disease is the most common cause of functional tricuspid regurgitation (FTR) in humans

Received February 10, 2021; received in revised form March 17, 2021; accepted March 18, 2021.

Correspondence: Xiao-fu Dai, Department of Cardiovascular Surgery, Union Hospital, Fujian Medical University, Fuzhou, 350001 People's Republic of China (e-mail: gzlde0323@msn.com).
[Dreyfus 2005]. Previous studies have shown that the prevalence of FTR in patients undergoing mitral valve surgery ranges from $8 \%$ to 45\% [Cohen 1987; Di Mauro 2013; Izumi 2002]. Over the years, cases of late FTR after isolated mitral valve surgery have been reported, causing considerable morbidity and mortality in these patients [Izumi 2002; King 1984; McCartney 2019].

In terms of the surgical indications for tricuspid regurgitation (TR), while the general view on left-sided valve disease combined with severe FTR requiring surgical intervention is the same, indication for concomitant TAP at the time of mitral valve surgery (MVS) in patients without severe TR is ambiguous. According to recent research, more aggressive interventional strategies are needed because of the low event-free survival and dismal prognosis of FTR after the progression of symptoms [Bianchi 2009]. A study focused on the prognosis of patients with mild to moderate TR during MVS also supported this view [Kara 2015]. In summary, the prevailing view is that an aggressive application of tricuspid annuloplasty can achieve better clinical outcomes for patients undergoing MVS [Dreyfus 2005; Takano 2017; De Meester 2015; Pfannmueller 2013; Calafiore 2009].

The 2017 valvular heart disease guidelines recommend that treatment for FTR should be performed early to avoid irreversible right ventricular dysfunction, and TAP should be performed at the same time of left heart valve surgery [Baumgartner 2017].

Although most of the appellate studies have been completed in the context of median sternotomy open heart surgery, the field of minimally invasive cardiac surgery has rapidly been developing over the past two decades. Previous studies have shown that minimally invasive cardiac surgery has significant benefits for patients, such as no disruption of the sternum structure, significantly relieved postoperative pain, reduced intensive care unit length of stay and postoperative hospital stay, and improved appearance of surgical incisions [Ritwick 2013]. However, there were few studies focusing on concomitant TAP in minimally invasive mitral valve surgery (MIMVS). Besides, the clinical outcomes of patients undergoing concomitant TAP during the MIMVS remain unclear.

Therefore, we conducted a retrospective analysis of patients who underwent totally endoscopic mitral valve surgery with concomitant tricuspid annuloplasty in our institution from January 2019 to June 2020. The propensity score matching (PSM) was performed to compare a minimally 
Table 1. Demographic and preoperative data compared between the two groups

\begin{tabular}{|c|c|c|c|c|c|c|}
\hline Age (years) & $60.52 \pm 9.97$ & $57.25 \pm 9.21$ & 0.046 & $58.29 \pm 10.75$ & $57.25 \pm 9.21$ & .601 \\
\hline $\mathrm{BMI}\left(\mathrm{kg} / \mathrm{m}^{2}\right)$ & $22.58 \pm 1.73$ & $22.46 \pm 1.56$ & 0.671 & $22.76 \pm 1.70$ & $22.46 \pm 1.56$ & .344 \\
\hline Atrial fibrillation & 48 & 22 & 0.643 & 22 & 22 & 1 \\
\hline NYHA class (median) & ॥ & ॥ & 0.975 & ॥ & $\|$ & .882 \\
\hline $\operatorname{LVED}(\mathrm{mm})$ & $57.80 \pm 8.57$ & $58.29 \pm 7.95$ & 0.726 & $58.55 \pm 8.46$ & $58.29 \pm 7.95$ & .876 \\
\hline LVEF (\%) & $57.07 \pm 5.41$ & $56.02 \pm 5.57$ & 0.250 & $55.56 \pm 5.49$ & $56.02 \pm 5.57$ & .676 \\
\hline SPAP $(\mathrm{mmHg})$ & $45.07 \pm 13.23$ & $44.84 \pm 16.38$ & 0.923 & $47.43 \pm 13.31$ & $44.84 \pm 16.38$ & .383 \\
\hline Mix lesion & 34 & 12 & - & 12 & 12 & \\
\hline TR grade & - & - & 0.930 & - & - & .787 \\
\hline 1 & 6 & 3 & - & 4 & 3 & \\
\hline 2 & 40 & 15 & - & 12 & 15 & \\
\hline 3 & 66 & 28 & - & 27 & 28 & \\
\hline 4 & 10 & 5 & - & 8 & 5 & \\
\hline
\end{tabular}

BMI, body mass index; NYHA class: New York Heart Association functional classification; LVED, left ventricular end diastolic diameter; LVEF, left ventricular ejection fraction; SPAP, pulmonary artery systolic pressure

invasive approach with median sternotomy approach tricuspid annuloplasty. We also aimed to evaluate the relevant surgical parameters, surgery-related complications, short-term survival rates, and to explore the advantages and disadvantages of concomitant tricuspid annuloplasty in patients undergoing totally endoscopic mitral valve surgery.

\section{MATERIALS AND METHODS}

\section{Patients}

We retrospectively analyzed the clinical data of the patients who visited our department from January 2019 to June 2020 to undergo concomitant tricuspid annuloplasty, during mitral valve surgery via a totally endoscopic approach or median sternotomy. All the participants had mitral valve disease and FTR, which can be treated with surgery under thoracoscopic guidance. All patients had been given alternative surgical treatment options during the preoperative interviews. The inclusion criteria were as follows: (1) Primary mitral valve disease with functional tricuspid regurgitation; and (2) No prior cardiac surgery or right thoracic surgery. The exclusion criteria were as follows: (1) Organic tricuspid regurgitation; (2) Severe peripheral arterial disease in lower limbs; (3) Severe cardiac insufficiency $(\mathrm{EF}<35 \%)$; (4) Thoracic deformity; (5)
Conversion to median sternotomy during totally endoscopic cardiac surgery; and (6) Requires concomitant aortic valve surgery and/or coronary artery bypass grafting.

\section{Echocardiography}

All patients underwent a transthoracic or transesophageal two-dimensional B-mode, M-mode, and Doppler echocardiography before operation to measure cardiac dimensions and the severity of valve regurgitation as recommended by the American Society of Echocardiography. The tricuspid annulus diameter and the severity of TR were assessed, according to current standard guidelines, and were graded on a scale from 1 to 4 (1=mild, $2=$ moderate, $3=$ moderate to severe, 4=severe) [Baumgartner 2017; Zoghbi 2003; Lang 2005; Quiñones 2002].

The indications for tricuspid annuloplasty were as follows: 1) moderate TR on preoperative echocardiography; 2) significant annular dilation assessed on pre-bypass echocardiography at end-diastolic diameter in the four-chamber view as an annular $>40 \mathrm{~mm}$; or 3 ) in unspecified cases, on the basis of direct assessment, intraoperative saline testing, and comparison of the anterior and posterior leaflet surface areas with the annulus size [Carpentier 2010]. Whether or not to perform TAP was affected by the degree of TR, tricuspid annular dimension and ventricular function, but ultimately was judged 
Table 2. Intra-operative data compared between two groups after PSM

\begin{tabular}{|c|c|c|c|}
\hline Item & MVS-TAP & MIMVS-TAP & $P$ \\
\hline Mitral valve surgery strategy & - & - & .219 \\
\hline Mitral valve replacement & 29 & 35 & \\
\hline Mitral valve repair & 22 & 16 & \\
\hline $\begin{array}{l}\text { Tricuspid valve surgery } \\
\text { strategy }\end{array}$ & - & - & .514 \\
\hline Kay & 0 & 2 & \\
\hline De Vega & 16 & 17 & \\
\hline Annuloplasty ring & 35 & 32 & \\
\hline $\begin{array}{l}\text { Annuloplasty ring size } \\
\text { (Median) }\end{array}$ & 28 & 28 & 1 \\
\hline Maze procedure & 7 & 4 & .338 \\
\hline $\begin{array}{l}\text { Overall procedure duration } \\
(\min )\end{array}$ & $210.31 \pm 25.64$ & $233.90 \pm 35.23$ & $<.001$ \\
\hline CPB time (min) & $129.00 \pm 20.67$ & $170.80 \pm 25.10$ & $<.001$ \\
\hline Aorta cross-clamp time (min) & $86.71 \pm 14.51$ & $111.63 \pm 16.77$ & $<.001$ \\
\hline
\end{tabular}

by the attending surgical surgeon. Because detailed tricuspid valve annular dimension was not routinely documented during the procedure, annular size was only considered an optional indicator for combination of tricuspid valve repair.

Operative mortality was defined as any death within 30 days or at any time, during the same hospital stay. Morbidity after heart valve interventions was defined, according to the guidelines published by Akins [Akins 2008]. Severe adverse events recorded in the present study included stroke, heart block, reintubation, new-onset renal failure, and surgical reexploration. This study was approved and monitored by the ethics committee of the Fujian Union Hospital. Informed consent was waived as it was a retrospective study.

\section{Surgical technique}

For patients undergoing totally endoscopic cardiac surgery, after general anesthesia with double-lumen endotracheal intubation, the patients were positioned in a supine position with the right chest elevated to $20^{\circ}$ to $30^{\circ}$. Femoral artery cannula and a single two-stage femoral venous cannula were used to establish cardiopulmonary bypass (CPB) through a $3-4 \mathrm{~cm}$ groin incision under transesophageal echocardiography guidance [Bonaros 2006].

The totally endoscopic procedure was performed via an endoscopic right minithoracotomy. Three ports in the right side of the chest wall were introduced for observation and operation [Ma 2011]. With the help of the transthoracic clamp techniques, the ascending aorta was clamped [Murzi 1997]. Immediately after, the antegrade HTK solution was applied for cardioplegia, and the right atrium was opened. Consecutively, the stay sutures were placed to retract the margin of the right atrium, then set the femoral venous cannula in the proper position until the atrial septum clearly can be seen [Ma 2011; Huang 2020]. The mitral valve was performed through the transseptal approach. The left atrium was entered via the atrial septal incision, two sets of stay sutures were used to snare the margin of the atrial septum and were pulled out of the port and properly secured. After a direct assessment of the valve lesion, MIMVS was performed. Continuous sutures were used to close the atrial septal incision and tricuspid annuloplasty immediately was performed following this. TR was repaired by De Vega annuloplasty, Kay annuloplasty, or using a tricuspid annuloplasty ring (Edwards MC3 Tricuspid Annuloplasty Ring, Edwards Lifesciences, USA). Surgical techniques were determined, based on each surgeon's preference. After carefully de-airing of the heart, $\mathrm{CPB}$ was disconnected, and all incisions were closed under a transesophageal echocardiography evaluation.

For patients undergoing median sternotomy valve surgery, the patient was placed in the supine position with endotracheal intubation. Following median sternotomy, the ascending aorta canula was inserted into the aorta, and the bicaval or right atrial venous cannulas were used to establish CPB. After the aorta was clamped, the cardioplegia was performed with the HTK solution, and mitral valve surgery and tricuspid annuloplasty were performed afterward.

\section{Statistical analysis}

SPSS software (IBM SPSS 24.0, SPSS Inc) was used to conduct all analyses, and $P$-values $<.05$ were defined as statistically significant. PSM was performed to account for differences in patient characteristics at baseline to minimize selection bias. The propensity score for each patient was calculated by logistic regression with adjustment for nine key baseline variables, including gender, age, hypertension, atrial fibrillation, New York Heart Association classification, body mass index, left ventricular end diastolic diameter, left ventricular ejection fraction, and pulmonary artery systolic pressure. Nearest-neighbor matching with a 0.05 caliper width without replacement was conducted when matching patients $1: 1$. The propensity matching score was used to match 173 patients, and a total of 51 pairs (102 cases) successfully were matched.

All quantitative data with normal distribution was expressed as mean \pm standard deviation. For nonnormally distributed variables, the Mann-Whitney $U$ test was applied. The independent samples t-test or ANOVA were used for continuous variables. For categorical data, the chi-square test or Fisher's exact test was applied.

\section{RESULTS}

One hundred seventy-three consecutive patients were selected for this study, 51 of whom underwent cardiac surgery with the totally endoscopic approach (MIMVS-TAP group) and 122 of whom underwent surgery through a median sternotomy (MVS-TAP group).

1. Comparison results of baseline data between two groups before PSM. In terms of baseline characteristics collected from the medical records, the age of the MIMVS-TAP group was younger than that of the MVS-TAP group (57.25 
Table 3. Postoperative Data compared between two groups after PSM

\begin{tabular}{|c|c|c|c|}
\hline Item & MVS-TAP & MIMVS-TAP & $P$ \\
\hline Early mortality & 1 & 0 & 1 \\
\hline Valve-related events & 1 & 0 & 1 \\
\hline Embolism & 0 & 0 & NS \\
\hline Bleeding event & 1 & 1 & 1 \\
\hline Reintervention & 1 & 1 & 1 \\
\hline Reintubation & 1 & 1 & 1 \\
\hline Heart block & 0 & 1 & 1 \\
\hline Wound problem & 0 & 2 & .495 \\
\hline Pneumothorax & 0 & 3 & .243 \\
\hline Subcutaneous emphysema & 0 & 2 & .495 \\
\hline Stroke & 1 & 0 & 1 \\
\hline Renal failure & 2 & 1 & 1 \\
\hline $\operatorname{LVED}(\mathrm{mm})$ & $55.55 \pm 6.34$ & $55.94 \pm 6.83$ & .764 \\
\hline LVEF (\%) & $57.53 \pm 6.26$ & $57.63 \pm 6.95$ & .940 \\
\hline $\mathrm{SPAP}(\mathrm{mmHg})$ & $33.14 \pm 7.59$ & $33.53 \pm 7.41$ & .792 \\
\hline ICU stay (hours) & $18.51 \pm 3.00$ & $16.44 \pm 2.38$ & $<.001$ \\
\hline Postoperative hospital stay (days) & $7.02 \pm 1.07$ & $5.45 \pm 2.04$ & $<.001$ \\
\hline
\end{tabular}

LVED, left ventricular end diastolic diameter; LVEF, left ventricular ejection fraction; SPAP, pulmonary artery systolic pressure; ICU, intensive care unit

\pm 9.21 versus $60.52 \pm 9.97$ years, $P=.046)$, and there were more female patients in the MIMVS-TAP group (56 women, $45.90 \%$ versus 32 women, $62.75 \%, P=.043)$. There was no statistical significance between the two groups with respect to the rest characteristics, such as hypertension, atrial fibrillation, New York Heart Association classification, body mass index, left ventricular end diastolic diameter, left ventricular ejection fraction, and pulmonary artery systolic pressure. (Table 1) The difference in average age and gender distribution between the two groups might be due to that younger patients and women had higher aesthetic requirements and were more likely to tolerate longer surgical time, resulting in age and gender differences between the two groups.

Since the unmatched baseline demographic and clinical data between the two groups will definitely bias the comparison, the data evaluation has to be made according to age and gender distribution. Therefore, it is necessary to select patients with similar conditions for comparison between the two groups. In this case, PSM could achieve a statistical effect of approximately random assignation of patients between the two groups.

2. Comparison results of baseline data between two groups after PSM. The demographic characteristics and baseline clinical information were well matched between the two groups. The descriptive statistics for the study population are shown in Table 1. There were no significant differences in the baseline data and lesion type of mitral valve and TR grade between the two groups.

3.Comparison of perioperative outcomes between the two groups after PSM

There were no significant differences in mitral and tricuspid valve surgery strategies. In patients undergoing tricuspid surgery, the median annuloplasty ring size in two groups were both $28 \mathrm{~mm}(P=1)$. The concomitant maze procedure also was not significantly different between the two groups. But the total operation time $(210.31 \pm 25.64$ versus $233.90 \pm 35.23$ mins, $P<.001)$, CPB time $(129.00 \pm 20.67$ versus $170.80 \pm$ 25.10 mins, $P<.001)$ and aorta cross-clamp time $(86.71 \pm$ 14.51 versus $111.63 \pm 16.77$ mins, $P<.001$ ) of MIMVS-TAP group were significantly longer than those of the MVS-TAP group (Table 2).

The postoperative data is detailed in Table 3. Only one death among the patients was reported in the study. The patient in the MVS-TAP group received ECMO support, due to postoperative low cardiac output syndrome, and finally died of multiple organ failure. There were no significant differences observed in postoperative adverse events between the two groups.

There were no postoperative embolization events in the MVS-TAP group. Gastrointestinal bleeding occurred in one patient, due to anticoagulation in MVS-TAP group. An additional endotracheal re-intubation was performed in the intensive care unit on one patient, who underwent redo mitral valve replacement for severe mitral regurgitation and hemoglobinuria after mitral valve repair in the MVS-TAP group. Two patients suffered from postoperative acute renal failure, including the one who received re-operation mentioned above. There were no poor wound healing problems, subcutaneous emphysema, and pneumothorax in this group. One patient had a sudden loss of consciousness after surgery, and brain hemorrhage subsequently was confirmed by emergency computed tomography. The patient was transferred to the department of neurology for further treatment.

One patient in the MIMVS-TAP group underwent reexploration immediately after surgery to stop the bleeding from the incision site. A patient received endotracheal intubation again, due to low blood oxygen saturation in the intensive care unit after operation. During hospitalization, this patient developed acute renal insufficiency and was treated with hemodiafiltration and eventually recovered and was discharged from the hospital. In the MIMVS-TAP group, incisional hydrops was observed in one patient. One patient had lymphatic leakage in the inguinal incision, three patients had pneumothorax, and two patients had subcutaneous emphysema. One patient who underwent ablation for atrial fibrillation was diagnosed with temporary third-degree heart block, and the temporary pacemaker was implanted. Subsequent reexamination before hospital discharge revealed incomplete right bundle branch block and left bundle branch block, and the patient successfully was discharged after cutting off the pacemaker.

In terms of the lengths of intensive care unit stay (18.51 \pm 3.00 versus $16.44 \pm 2.38$ hours, $P<.001)$ and postoperative hospital stay $(7.02 \pm 1.07$ versus $5.45 \pm 2.04$ days, $P<$ 
.001), the MIMVS-TAP group was statistically shorter than the MVS-TAP group. In terms of cardiac structure and function, postoperative echocardiography showed no significant differences in left ventricular end-diastolic diameter, left ventricular ejection fraction, and pulmonary artery pressure. No statistical differences existed between the two groups for the incidence of conduction blocks and stroke.

\section{DISCUSSION}

According to the latest heart valve guidelines, for patients with FTR who need left-sided valve surgery, tricuspid annuloplasty should be considered in patients suffering from severe TR or mild-to-moderate TR with a tricuspid annular dilatation $\left(\geq 40 \mathrm{~mm}\right.$ or $\left.>21 \mathrm{~mm} / \mathrm{m}^{2}\right)$ or right heart failure. The guidelines state that tricuspid annuloplasty can be beneficial for mild, moderate, or severe FTR during mitral valve surgery for patients with a dilated tricuspid annulus [Baumgartner 2017; Nishimura 2014].

However, these guidelines have an insufficient focus on totally endoscopic cardiac surgery. Since the 1990s, minimally invasive technique rapidly has developed in the cardiovascular field and gradually expanded its indications. Early results showed that there were no significant differences in the incidence of immediate and long-term complications and longterm survival rates between endoscopic and conventional open-heart mitral valve surgeries [Ritwick 2013; Lucà 2013; Grossi 2001]. However, most of the studies were limited to a small number of patients, lacking large or multicenter studies. In addition, these studies did not specifically focus on mitral valve disease with FTR. It is widely known that minimally invasive cardiac surgery is complicated to operate, owing to limited operating space and usually requiring long operating time and special perfusion strategy. Due to the physical limitations of MIMVS, unique retrograde perfusion strategy and inadequate de-airing theoretically lead to a higher incidence of neurological complications, which were a primary concern. Therefore, many have questioned the potential damage to the neurological system in patients undergoing endoscopic cardiac surgery, as well as the increased operation time [Grossi 2012; Gammie 2010].

Video-assisted mitral and tricuspid valve surgery undoubtedly increases the complexity of the operation and the duration of CPB time. Therefore, we conducted a study that focuses on the clinical outcome of concomitant tricuspid annuloplasty in totally endoscopic mitral valve surgery.

In our study, we found that the MIMVS-TAP group was younger and mostly female. The reason why we need to match the propensity score is, after analyzing the population sample, we found that there were significant differences between the two groups in age and gender. It may be that young people and women are more concerned about the aesthetic appearance of the incision and tend to choose MIMVS.

The main finding of this study was that patients receiving MIMVS+TAP due to FTR had comparable (or equally good) short-term outcomes compared with patients receiving MVS+TAP via median sternotomy. In contrast with the concern that increased operative time and difficulty manipulate of the MIMVS may lead to increased incidence of cerebrovascular accidents and third-degree heart block, there were no differences in short-term mortality or sever events, but there were advantages in terms of lengths of intensive care unit and postoperative hospital stays. Our study demonstrated similar results in patients undergoing totally endoscopic and conventional mitral valve surgery combined with tricuspid annuloplasty by PSM analysis. Concomitant TAP with MIMVS can safely be performed without increasing early mortality and morbidity, including heart block and stroke.

One of the common questions is whether the minimally invasive tricuspid valve repair is as effective as the median surgery. We noted that there were no significant differences in left ventricular structure and function or pulmonary artery pressure between the two groups after surgery. We did not measure detailed right ventricular parameters, but previous studies have shown that TAP at MVS can reverse right ventricular remodelling and improve functional status, especially in patients with valvular dilatation [Baumgartner 2017; Chikwe 2015].

The concurrent TAP during MIMVS had an impact on operative time. We found significant increases in cross-clamp time and CPB time, which led to an increase of nearly a half hour in the total surgical time.

In general, our study stated that mortality and morbidity, such as stroke and conduction block rates in MIMVS were not significantly different compared with median surgery after PSM. In addition, our research highlighted that minimally invasive cardiac surgery has great advantages over traditional median sternotomy due to its non-destruction of sternal structure, quality of life and aesthetic appearance of the incision [Huang 2020; Modi 2008; Iribarne 2012].

Despite these promising results, our study still has some limitations. First, this study only analyzed the totally endoscopic approach, which is only one of the minimally invasive cardiac surgery methods. Currently, for the mitral valve, there are several minimally invasive methods widely used, including lower hemisternotomy, direct-vision right minithoracotomy, endoscopic right minithoracotomy, and robotic-assisted right minithoracotomy. Which surgical approach is more appropriate for patients needs to be compared in multiple centers in order to develop surgical selection strategies based on treatment effectiveness and economic factors. Second, there are various techniques for tricuspid valve repair, and the main methods we use are tricuspid annuloplasty ring, De Vega, and kay. The long-term results of the different tricuspid valve repair techniques require longer-term follow-up studies. Third, the cohort was small, and the follow-up period was short. Long-term follow up is needed to clarify the longterm effects of concomitant TAP with MIMVS and whether concomitant TAP can improve the long-term prognosis of patients as well as median sternotomy [De Meester 2015; Chikwe 2015]. However, despite these limitations, our findings still provide new evidence for the selection of surgical approaches in clinical practice. 


\section{CONCLUSIONS}

Totally endoscopic mitral valve surgery with concomitant tricuspid annuloplasty is safe to perform, even with a long surgery duration. We found no difference in surgery-related complications. The only difference was that patients in MIMVS-TAP group had shorter intensive care unit stay and postoperative hospital stay than those receiving MVS-TAP.

\section{ACKNOWLEDGEMENTS AND DISCLOSURE}

We highly acknowledge the contribution by the participating doctors: Xue-shan Huang, Han-fan Qiu, and Dong-shan Liao. We declare that they have no competing interests. There is no financial support for this work.

\section{REFERENCES}

Akins CW, Miller DC, et al. 2008. Guidelines for reporting mortality and morbidity after cardiac valve interventions. Ann Thorac Surg. Apr; 85(4):1490-5.

Baumgartner H, Falk V, et al. 2017. 2017 ESC/EACTS guidelines for the management of valvular heart disease. Eur Heart J. 38(36):2739-91.

Bianchi G, Solinas M, et al. 2009. Which patient undergoing mitral valve surgery should also have the tricuspid repair? Interact Cardiovasc Thorac Surg. Dec; 9(6):1009-20.

Bonaros N, Schachner T, et al. 2006. Robotically assisted totally endoscopic atrial septal defect repair: insights from operative times, learning curves, and clinical outcome. Ann Thorac Surg. Aug;82(2):687-93.

Calafiore AM, Gallina S, et al. 2009. Mitral valve surgery for functional mitral regurgitation: should moderate-or-more tricuspid regurgitation be treated? A propensity score analysis. Ann Thorac Surg. 87(3):698-703.

Carpentier A, Adams DH, Filsoufi F. 2010. Carpentier's Reconstructive Valve Surgery. Saunders Elsevier, Philadelphia, PA, pp. 194-200.

Chikwe J, Itagaki S, et al. 2015. Impact of concomitant tricuspid Annuloplasty on tricuspid regurgitation, right ventricular function, and pulmonary artery hypertension after repair of mitral valve prolapse. J Am Coll Cardiol. 65(18):1931-8.

Cohen SR, Sell JE, et al. 1987. Tricuspid regurgitation in patients with acquired, chronic, pure mitral regurgitation. I. Prevalence, diagnosis, and comparison of preoperative clinical and hemodynamic features in patients with and without tricuspid regurgitation. J Thorac Cardiovasc Surg. Oct;94(4):481-7.

De Meester P, De Cock D, et al. 2015. Additional tricuspid annuloplasty in mitral valve surgery results in better clinical outcome. Heart. 101(9):720-6

Di Mauro M, Bezante GP, et al. 2013. Italian Study Group on Valvular Heart Disease Italian Society of Cardiology. Functional tricuspid regurgitation: an underestimated issue. Int J Cardiol. Sep 30;168(2):707-15.

Dreyfus GD, Corbi PJ, et al. 2005. Secondary tricuspid regurgitation or dilatation: which should be the criteria for surgical repair? Ann Thorac Surg. Jan;79(1):127-32.

Gammie JS, Zhao Y, et al. 2010. J. Maxwell Chamberlain Memorial Paper for adult cardiac surgery. Less-invasive mitral valve operations: trends and outcomes from the Society of Thoracic Surgeons Adult Cardiac Surgery Database. Ann Thorac Surg. Nov;90(5):1401-8, 1410.e1; discussion 1408-10

Grossi EA, LaPietra A, et al. 2001. Minimally invasive versus sternotomy approaches for mitral reconstruction: comparison of intermediate-term results. J Thorac Cardiovasc Surg. Apr;121(4):708-13.

Grossi EA, Loulmet DF, et al. 2012. Evolution of operative techniques and perfusion strategies for minimally invasive mitral valve repair. J Thorac Cardiovasc Surg. Apr;143(4 Suppl):S68-70.

Huang LC, Chen DZ, et al. 2020. Health-related quality of life following minimally invasive totally endoscopic mitral valve surgery. J Cardiothorac Surg. Jul 28;15(1):194.

Iribarne A, Easterwood R, et al. 2012. Comparative effectiveness of minimally invasive versus traditional sternotomy mitral valve surgery in elderly patients. J Thorac Cardiovasc Surg. Apr;143(4 Suppl):S86-90.

Izumi C, Iga K, Konishi T. 2002. Progression of isolated tricuspid regurgitation late after mitral valve surgery for rheumatic mitral valve disease. J Heart Valve Dis. May;11(3):353-6.

Kara I, Koksal C, et al. 2015. Outcomes of mild to moderate functional tricuspid regurgitation in patients undergoing mitral valve operations: a meta-analysis of 2,488 patients. Ann Thorac Surg. 100(6):2398-407.

King RM, Schaff HV, et al. 1984. Surgery for tricuspid regurgitation late after mitral valve replacement. Circulation. Sep;70(3 Pt 2):I193-7.

Lang RM, Bierig M, et al. 2005. Recommendations for chamber quantification: a report from the American Society of Echocardiography's Guidelines and Standards Committee and the Chamber Quantification Writing Group, developed in conjunction with the European Association of Echocardiography, a branch of the European Society of Cardiology. J Am Soc Echocardiogr. Dec;18(12):1440-63.

Lucà F, van Garsse L, et al. 2013. Minimally invasive mitral valve surgery: a systematic review. Minim Invasive Surg. 179569.

Ma ZS, Dong MF, et al. 2011. Totally thoracoscopic repair of atrial septal defect without robotic assistance: a single-center experience. J Thorac Cardiovasc Surg. Jun;141(6):1380-3.

McCartney SL, Taylor BS, Nicoara A. 2019. Functional Tricuspid Regurgitation in Mitral Valve Disease. Semin Cardiothorac Vasc Anesth. Mar;23(1):108-122

Modi P, Hassan A, Chitwood WR Jr. 2008. Minimally invasive mitral valve surgery: a systematic review and meta-analysis. Eur J Cardiothorac Surg. Nov;34(5):943-52.

Murzi M, Kallushi E, et al. 1997. Minimally Invasive Mitral Valve Repair Using Transthoracic Aortic Occlusion. Ann Thorac Surg. May;63(5):1477-9.

Nishimura RA, Otto CM, et al. 2014. 2014 AHA/ACC Guideline for the Management of Patients with Valvular Heart Disease: a report of the American College of Cardiology/American Heart Association Task Force on Practice Guidelines. Circulation. Jun 10;129(23):e521-643.

Pfannmueller B, Verevkin A, et al. 2013. Role of tricuspid valve repair for moderate tricuspid regurgitation during minimally invasive mitral valve surgery. Thorac Cardiovasc Surg. 61(5):386-91.

Quiñones MA, Otto CM, et al. 2002. Recommendations for quantification of Doppler echocardiography: a report from the Doppler Quantification Task Force of the Nomenclature and Standards Committee of the American Society of Echocardiography. J Am Soc Echocardiogr. Feb;15(2):167-84.

Ritwick B, Chaudhuri K, et al. 2013. Minimally invasive mitral valve 
procedures: the current state. Minim Invasive Surg. 679276.

Takano H, Hiramatsu M, et al. 2017. Severe tricuspid regurgitation after mitral valve surgery: the risk factors and results of the aggressive application of prophylactic tricuspid valve repair. Surg Today 47, 445-456.
Zoghbi WA, Enriquez-Sarano M, et al. 2003. American Society of Echocardiography. Recommendations for evaluation of the severity of native valvular regurgitation with two-dimensional and Doppler echocardiography. J Am Soc Echocardiogr. Jul;16(7):777-802. 